\title{
Feasibility of an Educational Program for Public Health Nurses to Promote Local Healthcare Planning: Protocol for a Pilot Randomized Controlled Trial
}

Kyoko Yoshioka-Maeda ( $\nabla$ yoshioka.k.aa@niph.go.jp)

National Institute of Public Health: Kokuritsu Hoken Iryo Kagakuin https://orcid.org/0000-0003-03440143

\section{Takafumi Katayama}

Hyogo Kenritsu Daigaku

Misa Shiomi

Kyoto University: Kyoto Daigaku

Noriko Hosoya

Chiba Kenritsu Hoken Iryo Daigaku

Hitoshi Fujii

Mejiro University: Mejiro Daigaku

Tatsushi Mayama

Doshisha Daigaku - Imadegawa Campus: Doshisha Daigaku

\section{Study Protocol}

Keywords: competency, continuing education, local healthcare planning, public health nursing, randomized controlled trial, web-based education

Posted Date: April 1st, 2021

DOl: https://doi.org/10.21203/rs.3.rs-368493/v1

License: (c) (i) This work is licensed under a Creative Commons Attribution 4.0 International License. Read Full License 


\section{Abstract}

Background: The promotion of local healthcare planning is crucial to assist public health nurses in improving community health inequities. However, there is no effective educational program for developing relevant skills and knowledge among these nurses. Therefore, this study aims to assess the feasibility and acceptability of a newly developed web-based self-learning program, designed to promote the involvement of public health nurses in the local healthcare planning process.

Methods: This pilot randomized control trial will randomly allocate eligible public health nurses to intervention and control wait-list groups [1:1]. The intervention group will be exposed to six web-based learning modules from July to October 2021. After collecting post-test data, the wait-list group will be exposed to the same modules to ensure learning equity. We will then evaluate the primary outcomes by implementing a validated and standardized scale designed to measure public health policy competencies both at baseline and post-intervention, while secondary outcomes will be measured on an action scale to demonstrate the necessity of healthcare activities. The third outcome will be knowledge and skills related to local healthcare planning by public health nurses. Participants will also provide feedback on both trial feasibility and the web-based self-learning program itself, which will help us identify improvement points for continual refinement. Feedback will be given through free descriptions.

Discussion: This pilot study will assess the feasibility and preliminary effects of a web-based selflearning program designed to develop the competencies of public health nurses involved in local healthcare planning. We will examine all outcome scales and data collection procedures in preparation for a future definitive randomized controlled trial. This will provide preliminary data for an intervention aimed at improving relevant competencies among public health nurses who are tasked with resolving health inequities in their respective communities through local health planning.

Trial registration: The protocol for this study was registered with the University Hospital Medical Information Network Clinical Trials Registry and approved by the International Committee of Medical Journal Editors (No. UMIN000043628, March 23, 2021).

\section{Background}

Health inequities and community health are important areas of focus across the world. In this regard, public health policies should target health gaps while aiming to resolve current and future community health needs [1]. To reduce vulnerabilities and address the unequal consequences of illness, interventions should focus on the social determinants of health, such as the social gradient, social exclusions, and social support, which are the root causes of health inequities [2]. In particular, local governments must aim to develop their own public health policies and effective inter-sectoral programs [3]. In the international context, public health nurses (PHNs) are responsible for improving a variety of health inequities, such as lack of limited access to healthcare services [4], as their unique professional roles provide a way to directly identify and address specific community health needs [5]. Local healthcare 
planning is a crucial strategy that PHNs can implement to reduce health inequities and bridge the gaps between community health needs [6].

PHNs may face substantial challenges when attempting to develop competencies that are relevant to local healthcare planning $[6,7]$. A previous systematic review investigated the necessary competencies that must be developed in order to implement local healthcare planning, such as using narratives [8], while other studies have focused on specific competencies related to development of health programs, such as determining needed programs and building consensus among those concerned $[9,10]$ and relevant practical methodologies, such as understanding policy process and securing budget $[11,12]$. Since needs-oriented health policies are considered areas in which PHNs hold tacit knowledge, it is important to provide avenues to share and utilize this knowledge in the context of human resources development, enhancing competencies among junior PHNs [13]. In this regard, continued education should be implemented to promote the competencies and self-confidence PHNs need to develop local healthcare planning competencies $[13,14]$. Recently studies have demonstrated that web-based learning programs, which enable recipients to engage from locations that do not require traditional face-to-face group sessions, provide several advantages, including user-friendly interfaces that are flexible, convenient, and time saving $[15,16]$. In fact, research has shown that web-based programs are just as effective for developing competencies as face-to-face methods $[17,18]$. However, there have been no randomized controlled trials (RCT) in this area, meaning, little is currently known about the effectiveness of web-based learning specifically targeted at PHNs $[12,16]$.

In Japan, a national licensing system has been introduced to qualify PHNs, approximately $60 \%$ of whom work for prefectural or municipal governments [19]. In this context, PHNs are expected to remain involved in the local healthcare planning process. Indeed, the Ministry of Health, Labour and Welfare published practical guidelines for PHNs in 2013, increasing their expected level of involvement in local healthcare planning. The guidelines specifically outlined their responsibilities to resolve present and future community health needs while developing local healthcare plans; these are now considered essential areas of competency for PHNs [20]. In fact, undergraduate curricula began to include these skills in 2007 [21], and the national PHN examination added related requirements in 2017 [22]. Even though many PHNs learn these competencies in their undergraduate course, there is a lack of continued education opportunities relevant to local healthcare planning [23]. Previous studies have not investigated whether PHNs who focused on self-directed learning improved self-confidence and competencies [24] and selfdirected learning readiness [25]. Although each local government should provide on-the-job training for PHNs, reports have indicated that approximately only half actually receive this type of continued education [26].

Further, Japan is facing declining populations due to the falling birthrate and aging population. Consequently, local governments are promoting the reduction of public service positions despite the necessity of needs-oriented local healthcare plans [27]. The COVID-19 pandemic has also accelerated the growing shortage of PHNs [28], which emphasizes the need for effective and efficient educational programs, especially those targeted at improving competencies related to local healthcare planning. 
Thus, a web-based learning program would help PHNs understand theoretical concepts and practical strategies that are relevant to the healthcare planning process. This will also enable them to access information at any time without the need to make contact with other participants. As such, this study will verify the effects/feasibility of a user-friendly web-based self-learning program designed to improve competencies for PHNs who are involved in the local healthcare planning process.

\section{Aim}

This pilot study aims to assess the feasibility and acceptability of a newly developed web-based selflearning program designed for PHNs who are involved in local healthcare planning. Further, the study will verify the program outcomes and data collection methods through relevant scales, and examine the preliminary effects of the web-based self-learning program, thus providing relevant data for calculating the sample size needed to conduct a future definitive RCT.

\section{Methods}

\section{Trial design and settings}

We will implement a pilot prospective RCT design among single-blind and parallel-groups. PHNs who register for this study will be randomly allocated to the intervention or wait-listed control groups [1:1]; as such, a double-blind design was not possible. However, we will ensure equal learning opportunities for both groups following data collection. All PHNs will be exposed to the same self-learning program. The intervention group will participate in the program first and then the wait-listed control group will be offered the same program contents. All participants will answer a post-test survey.

\section{Ethical considerations}

The Institutional Review Board (IRB) at the organization affiliated with the primary researcher approved of the protocol for this study on February 17, 2021 (ID: NIPH-IBRA\#12313). The IRB of the third researcher also approved of the study protocol on March 16, 2021 (ID: C1516).

This pilot study was registered with the University Hospital Medical Information Network Clinical Trials Registry (UMIN-CTR) following the International Committee of Medical Journal Editors (No. UMIN000043628, March 23, 2021). Explanations of the study's aims and procedures will be provided to the participants through the study website, where the results will also be published. All eligible PHNs will provide written web-based informed consent prior to the study registration. Only those who meet the inclusion criteria and agreed to participate will be included in the study. To ensure privacy, no personal information, including place of employment, will be collected.

\section{Participant eligibility criteria}

Eligible participants will include PHNs who are full-time public servants in Japan. PHNs who have experience in local healthcare planning will not be excluded. Exclusion criteria include those who do not 
have Internet access, are employed on a part-time basis, and have no opportunity to be involved in the local healthcare planning process.

\section{Sample size}

The sample size has not yet been calculated based on a power analysis due to feasibility and a limited research budget [29]. Further, previous research has indicated that sample size do not need to be calculated for pilot studies [30]. Owing to ethical considerations, we will not over-recruitment participants. Further, we expect the participant pool to be limited due to the COVID-19 pandemic, which has resulted in a general shortage of PHNs because most were involved in frontline activities [28].

\section{Sampling, informed consent and randomization}

In this pilot study, we will focus on a prefecture in the Kanto region in Japan, which has approximately 600 PHNs. The research team will send 36 letters (i.e., one to the prefectural government and 35 to the municipal governments) to public health nursing directors in May 2021. We will send a reminder letter to them in early June 2021. These directors are expected to share information about this study with their PHNs. Those who express interest in participation will be given access to our study website, which will contain a study explanation sheet and written web-based informed consent forms for completion. Only those who agree to these conditions and meet the eligibility criteria (as determined by the researchers) will be allowed to participate. The registration period will begin in May and last until the end of June 2021. To ensure privacy, participants will register using pseudonyms and their e-mail addresses.

Figure 1 illustrates a flowchart for the Consolidate Standards of Reporting Trial (CONSORT) design, which will be used throughout the study process. Randomization will be conducted by the researcher who specializes in statistics, and the co-author, via an equal computer-based randomization table. A permuted-block method will be adopted to ensure that participants are equally divided between the intervention and control groups. We will send e-mail to all participants informing them of which group they have been assigned to allocate.

\section{Minimizing bias}

All data will be anonymized; we will not collect any personal information or the names of the local governments where participants are employed. Data collection will be conducted through the study website, where participants will enter all relevant information and complete any required elements (e.g., demographic data and the outcome scales), which will be evaluated as baseline and post-test data. The intervention group will also be asked to keep the contents of the web-based self-learning program confidential so that the control group will not be exposed to any such information. We will explain the risks of contamination to the intervention group on the study website. The intervention group will receive a URL address where they can view the learning modules, which will be password protected (passwords sent via e-mail). 
We designed an outline of the web-based self-learning program, which included six modules for acquiring knowledge and skills related to local healthcare planning based on the adult learning theory [31], a review of the relevant Japanese literature [13], and several specific studies [5, 15, 16, 20, 23, 26, 32]. In the pretest, we asked five PHNs with previous involvement in local healthcare planning to confirm all modules and its contents. We then added explanations for each module and confirmed that the newly developed web-based self-learning program would include knowledge and skills that PHNs could use to promote local healthcare planning both effortlessly and effectively. Each module takes approximately $10-20$ minutes to complete.

Table 1 presents the themes and objectives for each module as follows: (1) Create a concrete vision of the ideal community; (2) Bridge the daily practices of PHNs' with local healthcare planning; (3) Basic knowledge necessary for local healthcare planning; (4) Understand the steps of the healthcare planning process; (5) Hone coordinate skills with related parties; and (6) Accomplish goal setting and project evaluation. Participants will be able to use this worksheet to identify and summarize the objectives for local healthcare planning, community health needs, related government plans, legal issues, relevant staff and key persons in the community, and an evaluation index. The intervention will be conducted from July to August 2021. Once the post-test data are collected in September, the control group will participate in the program from October to November in 2021.

\section{Outcomes and data collection}

Using the study website, eligible participants will provide demographic data and complete the outcome scales both at baseline and post-intervention. More specifically, demographic data will include gender, age, years of PHN experience, job title, educational background, affiliation, previous experience with local healthcare planning education at the undergraduate level and/or on-the-job training after graduation, previous experience with local healthcare planning, whether they worked with colleagues who played active roles in healthcare planning, and information on promoting the daily practices of PHNs. To decrease the number of dropouts, we will send e-mails all participants with information about the study progress and remind them to answer the post-test questions.

Both at baseline and post-intervention, we will obtain participants data and investigate the following outcomes.

\section{Competencies related to involvement in health policy}

The primary outcome will be measured using a self-reported 16-item scale targeting PHN competencies in public health policy in the context of the Japanese local governments [33]. The scale was confirmed as both reliable and valid for use in Japan and is only available in Japanese. The first eight items measure community partnership among PHNs when developing health policy, while the remaining items evaluate competencies related to the community nursing diagnosis cycle. All items will be measured using a 4point Likert scale, ranging from 0 to 3: 0 (unable to do), 1 (partially unable to do), 2 (partially able to do), and 3 (able to do). 


\section{The necessity of healthcare activities}

The secondary outcome will be measured using a self-reported 19 item scale designed to reveal information about the necessity of healthcare activities that are based on evidence and the promotion of decision-making with superiors as a way to improve planning [34]. This scale was confirmed as both reliable and valid for use in Japan and is only available in Japanese. The 19 items listed in the scale are organized within four dimensions as follows: 1) three items measured the existence of health needs, 2) five items measure the necessity of addressing health needs based on evidence, 3) five items measure actual conditions that require solutions, and 4) six items measure priorities for resolution. All items will be measured using a 6-point Likert scale: 0 (never applicable), 1 (20\% applicable), 2 (40\% applicable), 3 (60\% applicable), 4 (80\% applicable), and 5 (100\% applicable).

\section{Knowledges, skills, and perspectives of PHNs involved in local healthcare planning}

The third outcome will be measured based on 29 questions targeting the knowledge, skills, and perspectives of PHNs involved in local healthcare planning. All items will be measured using a 4-point Likert scale: 1 (never), 2 (not much), 3 (a little), and 4 (a lot).

\section{Trial feasibility}

We will assess the feasibility of this study based on the recruitment rate, dropout rate, and data completion rate. We will also ask the participants for their feedback on the feasibility of the trial and the web-based learning self-learning program itself, clarifying areas that need improvement and/or continual refinement. These responses will be obtained through free descriptions.

\section{Data management}

Without harmful interventions, this study will not establish a data monitoring committee. Researcher TK will be responsible for managing all data obtained through the study website, and will alert the research team about any problems related to recruitment or data collection. Researcher TK will also provide all data to the research team via Microsoft Excel (password locked). The research team will also conduct a quality assurance procedure.

\section{Statistical analysis}

We will conduct descriptive statistics (e.g., recruitment rate, dropout rate, and data completion rate) and analyze intergroup baseline differences in the data and primary outcomes using the Mann-Whitney U-test and the chi-square/Fisher's exact test. Next, we will assess whether previous experience in local healthcare planning and other factors affected the study outcomes based on the results of the MannWhitney U-test and the chi-square/Fisher's exact test. Analyses will be performed according to the intention-to-treat principle. We will use IBM SPSS for Windows (version 25; IBM Corp, Armonk, NY, USA) for analysis and $p$-values $<.05$ indicating statistically significant intergroup differences. This study is expected to have a power $\geq .80$ and $a \leq .05$, assuming a medium effect size $(d=.30)$ [35]. 


\section{A content analysis}

We will conduct a content analysis using all participant feedback provided through the free descriptions mentioned earlier, specifically regarding their feasibility and the web-based self-learning program itself. This will be done by entering relevant information into a Microsoft Excel worksheet. We will code all data and sort the results based on their commonalities. These findings will be used to refine both the study website and the web-based learning self-learning program.

\section{Discussion}

This pilot randomized controlled trial will aim to confirm both the feasibility and preliminary effects of a web-based self-learning program designed to increase the involvement of PHNs in local healthcare planning by enhancing relevant competencies. We will also examine the eligibility of the implemented outcome scales and data collection procedures in preparation for a future definitive RCT. The strengths of the novel self-learning program are as follows: (1) user-friendly web-based educational program, (2) eligible participants will be selected based on clear criteria, (3) all participants will be given equal opportunities to learn about local healthcare planning through the program (i.e., after we collect data from the intervention group), and (4) primary outcomes will be measured using validated standardized scales.

We developed the abovementioned program based on several components, including adult learning theory [31], a nationwide survey [24], and previous related studies [5, 15, 16, 20, 23, 24]. Through participation in web-based self-learning modules, the participants will acquire the competencies of local healthcare planning and bridge the gaps between the community health needs that PHNs must address both in daily practice and in the context of newly developed local healthcare plans. Development of local healthcare planning can improve health inequities by bridging the gaps in community health needs [6]. In this regard, PHNs are responsible for enhancing health equity and developing needs-oriented health policy through their practices [4, 5]. This pilot feasibility study will also adopt validated outcome scales, which will be used to gather the preliminary data needed to calculate an appropriate sample size for a future large-scale RCT. Owing to the constraints of the COVID-19 pandemic, we will also ensure that participants are socially distanced to prevent infection. More specifically, our web-based learning modules do not require participants to make direct contact with one another and will also eliminate travel time.

As mentioned, participants in the control group will be able to access the same learning program in the post-intervention context, providing them with critical education while minimizing the number of dropouts and ensuring educational equality. Further, we will assess whether previous experience with local healthcare planning impacts the study outcomes. These results will be used to revise different aspects of the program as well as the inclusion criteria for a future definitive RCT.

We have also identified some potential limitations. First, the pilot study will only include a small sample from one prefecture in Japan. In this regard, participants will likely have strong interests in the nature of 
the study program, thus increasing their motivation to engage in continued education. This may result in selection bias and/or influence the distribution of data. However, this study will focus on the feasibility and acceptability of the program, not its effectiveness. Regardless, these issues should be carefully considered when interpreting the data. Second, we will protect the privacy of all participants by anonymizing the data, and will not collect the names of the local governments where they are employed. As such, we cannot rule out the possibility that eligible participants from the same local governments will be allocated to different study groups. In this regard, we cannot entirely prevent contamination or instances in which the program contents are leaked from the intervention group to the wait-listed control group. We will explain this issue to all participants, and repeatedly ask the intervention group to keep the program contents confidential. Third, we could not control some confounding factors, such as the provenance area of participants and rural vs. urban areas, and the lack of follow-up assessment in this study. Fourth, only five PHNs confirmed the web-based self-learning modules due to the COVID-19 pandemic. Fifth, the reliability and validity of the Japanese version of Fisher's Self-Directed Learning Readiness Scale has not been confirmed. Thus, we could not use this scale in this study.

Despite these limitations, this pilot RCT will assess the feasibility and acceptability of a newly developed web-based self-learning program designed to increase the involvement of PHNs in local healthcare planning while also providing the preliminary data needed for a future definitive RCT in Japan. Therefore, we will confirm whether the web-based, self-learning system is considered user-friendly and whether participants exhibit improved competencies in the local healthcare planning context following the intervention.

\section{Conclusions}

This pilot RCT will determine the feasibility of a novel web-based, self-learning program for PHNs in local healthcare planning. We will also confirm the appropriateness of the enrollment process and data collection procedures. Further, the results of the pilot study will provide preliminary data and essential feedback for use in a future main RCT. Here, the challenge is to provide new evidence for improving competencies among PHNs who are tasked with resolving health inequities in their respective communities via local healthcare planning.

\section{Trial Status}

Trial registration: No. UMIN000043628, Registered March 23, 2021. Enrolling and date recruitment began: May/2021. Approximate date recruitment will be completed: June 30, 2021. Latest protocol version approved February 17, 2021, National Institute of Public Health and 2021 (NIPH-IRBA\#12313), and approved March 16, 2021, Graduate School of Medicine, Kyoto University (C1516). Recruitment has not yet begun.

\section{Abbreviations}


IRB: institutional Review Board

PHNs: public health nurses

RCT: Randomized controlled trial

\section{Declarations}

\section{Ethics approval and consent to participate}

The IRB of the National Institute of Public Health provided approval on February 17, 2021 (reference no. NIPHIRB\#12313), and approved March 16, 2021, Graduate School of Medicine, Kyoto University (C1516) for the study. The IRB will be informed about the necessary updates in case the protocol is revised. This study will be conducted in accordance with the ethical standards of the Declaration of Helsinki. We will also develop a website that provides all participants with an explanation of this study purpose and procedures and publish the study results after completion. All eligible participants will be required to sign written web-based written informed consent forms prior to the study registration.

\section{Consent for publication}

Not applicable.

\section{Availability of data and materials}

Not applicable.

\section{Competing interests}

The authors declare that they have no competing interests.

\section{Funding}

This work was supported by JSPS KAKENHI Grant Number $19 \mathrm{H03972} \mathrm{(to} \mathrm{KYM).}$

\section{Authors' contributions}

KYM, TK, MS, NH, HF, and TM conceived and designed the study. KYM and TK developed the statistical analysis plan. KYM created the first draft of the manuscript. All authors edited the manuscript and read and approved the final draft.

\section{Acknowledgements}

We thank all five PHNs who supported our study. We also thank Editage (www. editage.com) for their writing support. 


\section{References}

1. Marmot M. The health gap: the challenge of an unequal world: the argument. Int J Epidemiol. 2017; 46(4): 1312-1318. https://doi: 10.1093/ije/dyx163.

2. World Health Organization. Conceptual framework for action on the social determinants of health: 2010. Available from:

https://www.who.int/sdhconference/resources/ConceptualframeworkforactiononSDH_eng.pdf. (accessed 22 March, 2021).

3. Lee J, Schram A, Riley E, Harris P, Baum F, Fisher M, Freeman T, Friel S. Addressing health equity through action on the social determinants of health: A global review of policy outcome evaluation methods. Int J Health Policy Manag. 2018; 7(7): 581-592. https://doi: 10.15171/ijhpm.2018.04.

4. The Public Health Nursing Section, American Public Health Association. The definition and practice of public health nursing: A statement of the public health nursing section. 2013. Available from: https://www.apha.org/ /media/files/pdf/membergroups/phn/nursingdefinition.ashx. (accessed 22 March, 2021).

5. Quad Council Coalition. Community/public health nursing competencies: 2018. Available from: https://www.cphno.org/wp-content/uploads/2020/08/QCC-C-PHN-COMPETENCIESApproved_2018.05.04_Final-002.pdf. (accessed 22 March, 2021).

6. Yoshioka-Maeda K: Promoting needs-oriented health programme planning through public health nurses in Japan. J Adv Nurs. 2020; 76(7): 1496-1497. https://doi.org/10.1111/jan.14337.

7. Polivka BJ, Chaudry RV. Public Health Nursing Position Descriptions Congruence with ANA Standards, Public Health Essential Services, and Quad Council Domains. Public Health Nurs. 2015; 32(5): 532-542. https://doi.org/10.1111/phn.12148.

8. Fadlallah R, El-Jardali F, Nomier M, Hemadi N, Arif K, Langlois EV, Akl EA. Using narratives to impact health policy-making: a systematic review. Health Res Policy Syst. 2019; 17(1): 26. https://doi.org/10.1186/s12961-019-0423-4.

9. Yoshioka-Maeda K, Murashima S: Difficulties and solutions related to the experience of developing a new project by Japanese municipal public health nurses according to their seniority. Nihon Koshu Eisei Zasshi. 2013; 60(1): 21-29. (in Japanese)

10. Campbell LA, Harmon MJ, Joyce BL, Little SH. Quad Council Coalition community/public health nursing competencies: Building consensus through collaboration. Public Health Nurs. 2019; 37(1): 96-112. https://doi.org/10.1111/phn.12666.

11. Heiman H, Smith L, McKool M, Mitchell D, Roth Bayer C: Health Policy Training: A Review of the Literature. Int J Environ Res Public Health. 2015; 13(1): ijerph13010020. https://doi: 10.3390/ijerph13010020.

12. Yoshioka-Maeda K, Shiomi M, Katayama T, Hosoya N, Kuroda M. Effectiveness of an educational program for mid-level Japanese public health nurses to improve program planning competencies: A 
preliminary randomized control trial. Public Health Nurs. 2019; 36(3): 388-400.

https://doi.org/10.1111/phn.12580.

13. Yoshioka-Maeda K. A comprehensive review of related literatures focuses on the strategies of health, medical care and welfare planning by utilizing Japanese public health nurses (since 2013-2018). $J$ Japan Academy of Community Health Nurs 2020; 23: 59-65. (in Japanese)

14. Ogawa T, Nakatani $H$. Factors associated with professional confidence in Japanese public health nurses: A cross-sectional survey. Public Health Nurs. 2020; 37(2): 272-280. https://doi.org/10.1111/phn.12705.

15. Du S, Liu Z, Liu S, Yin H, Xu G, Zhang H, Wang A. Web-based distance learning for nurse education: a systematic review. Int Nurs Rev. 2013; 60(2): 167-177. https://doi.org/10.1111/inr.12015.

16. Yoshioka-Maeda K, Shiomi M, Katayama T, Hosoya N. Impact of web-based learning for health program planning competency, knowledge and skills among mid-level public health nurses: $\mathrm{A}$ randomized controlled trial. Public Health Nurs. 2019; 36(6): 836-846. https://doi.org/10.1111/phn.12642.

17. Lankshear S, Huckstep S, Lefebre N, Leiterman J, Simon D: The ALIVE program: developing a webbased professional development program for nursing leaders in the home healthcare sector. Nurs Leadersh. 2010; 23 (Spec No 2010): 61-74. https://doi: 10.12927/cjnl.2010.21747.

18. McCutcheon K, Lohan M, Traynor M, Martin D: A systematic review evaluating the impact of online or blended learning vs. face-to-face learning of clinical skills in undergraduate nurse education. $J$ Adv Nurs 2015; 71(2): 255-270. https://doi.org/10.1111/jan.12509.

19. Japanese Nursing Association. Nursing in Japan: 2016 Available from: https://www.nurse.or.jp/jna/english/pdf/nursing-in-japan2016.pdf. (accessed 22 March, 2021).

20. Japan Ministry of Health, Labour, and Law. Chiiki ni okeru hokenshi no hoken katudou ni tsuite [Guideline of health activities for public health nurses]: 2013. Available from:

https://www.mhlw.go.jp/web/t_doc?datald=00tb9310\&dataType=1\&pageNo=1. (accessed 22 March, 2021).

21. Japan Ministry of Health, Labour, and Law. Hokenshi kyoiku no gijutsu komoku to sotsugyoji no totatsudo [A framework for assessing essential public health nursing skills and achievement levels required for students graduating from schools]: 2008. Available from: https://www.mhlw.go.jp/shingi/2009/04/dl/s0428-8m.pdf. (accessed 22 March, 2021).

22. Japan Ministry of Health, Labour, and Law. Hokenshi Jyosanshi Kangoshi kokkashiken syutudai kijyun Heisei 30 nendo ban ni tsuite [Standards for the national examination for public health nurses, midwives, and nurses]: 2017. Available from: https://www.mhlw.go.jp/file/04-Houdouhappyou10803000-Iseikyoku-ljika/0000158962.pdf. (accessed 22 March, 2021).

23. Shiomi M. Evaluation of the educational program to enhance project creating abilities of mid-career public health nurses through the action research. Univ Hyogo, College of Nurs Art and Sci, Res Insti Nurs Care for People and Community bulletin. 2015; 22: 41-53 (in Japanese). 
24. Tekkol IA, Demirel M. An investigation of self-directed learning Skills of Undergraduate Students. Front Psychol. 2018; 9: 2324. https://doi.org/10.3389/fpsyg.2018.02324

25. Chakkaravarthy K, Ibrahim N, Mahmud M, Hardaker G, Venkatasalu MR. Determinants of readiness towards self-directed learning among nurses and midwives: Results from national survey. Nurse Educ Pract. 2020; 47: 102824. doi: 10.1016/j.nepr.2020.102824.

26. Yoshioka-Maeda K, Shiomi M, Katayama T, Hosoya N, Fujii H, Mayama T. Self-reported competences of public health nurses for developing needs-oriented local healthcare plans: A nationwide crosssectional survey. J Adv Nurs. 2021; Jan 10. https://doi.org/10.1111/jan.14741. (Online ahead of print)

27. Japan Ministry of Internal Affaires and Communications. Chihou koumuin suu no genjyou [The number of local public employees. 2020. Available from:

https://www.soumu.go.jp/main_content/000608432.pdf. (accessed 22 March, 2021).

28. Yoshioka-Maeda K, Iwasaki-Motegi R, Honda C. Preventing the dysfunction of public health centres responding to COVID-19 by focusing on public health nurses in Japan. $J$ Adv Nurs. 2020; 76(9): 2215-2216. https://doi.org/10.1111/jan.14409.

29. Shanyinde M, Pickering, R. M., Weatherall, M. Questions asked and answered in pilot and feasibility randomized controlled trials. BMC Med Res Methodol. 2011; 11: 117. https://doi: 10.1186/14712288-11-117.

30. Eldridge SM, Chan CL, Campbell MJ, Bond CM, Hopewell S, Thabane L, Lancaster GA, PAFS consensus group. CONSORT 2010 statement: extension to randomised pilot and feasibility trials. Pilot Feasibility Stud. 2016; 2: 64. https://doi: 10.1186/s40814-016-0105-8.

31. Knowles MS. The modern practice of adult education: From pedagogy to andragogy. New York: The Adult Education Company; 1980.

32. Saeki K, Hirano M, Honda H, Asahara K. Developing a comprehensive career development scale for public health nurses in Japan. Public Health Nurs. 2019: 37(1): 135-143. https://doi.org/10.1111/phn.12673.

33. Suzuki Y, Tadaka, E. Development of a scale measuring the competences of public health nurses in publichealth policy in Japanese administrative agencies. Nihon Koshu Eisei Zasshi. 2014; 61(6): 275-285. https://doi.org/10.11236/jph.61.6_275. (in Japanese)

34. Okamoto R, Hatono, Y., Koide, K., Nagano, F., Iwamoto, S.,Kusano, E. Development of an action scale to show the necessity of healthcare activities. Nihon Koshu Eisei Zasshi. 2015; 62(6): 271-280. https://doi.org/10.11236/jph.62.6_271. (in Japanese)

35. Cohen, J. A power primer. Psychological Bulletin. 1992; 1: 155-159. https://doi: 10.1037//00332909.112.1.155.

\section{Tables}

Due to technical limitations, table PDF is only available as a download in the Supplemental Files section. 


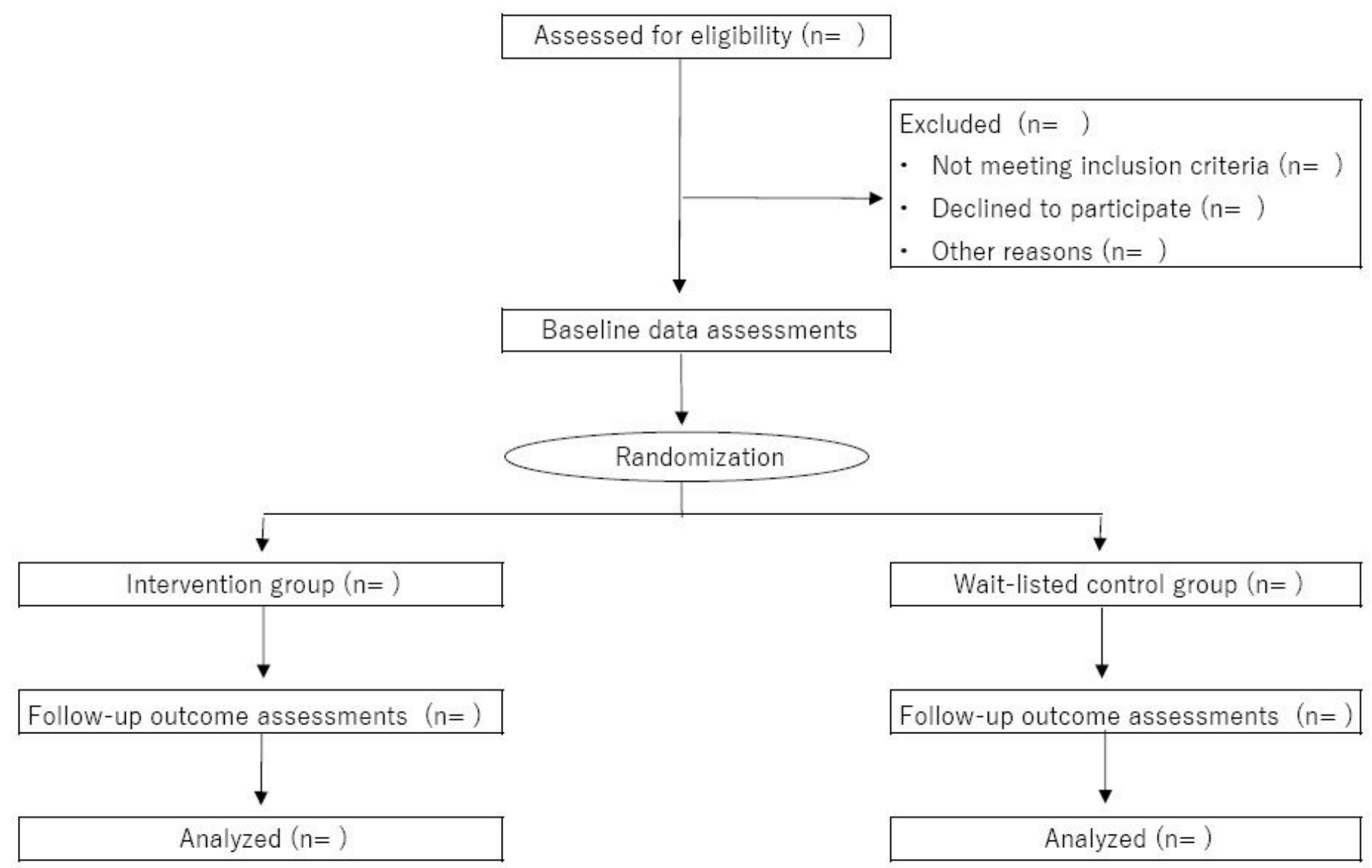

Figure 1

Consolidated Standards of Reporting Trial (CONSORT) flowchart

\section{Supplementary Files}

This is a list of supplementary files associated with this preprint. Click to download.

- Appendix210323.docx

- SPIRITFillablechecklist210323.doc

- Table1210323.pdf 